\title{
Animal Experiment
}

\section{Evaluation of Interaction between a Spinal Cord Stimulator and Implanted Cardioverter- Defibrillator in a Swine Model}

Gassan M. Chaiban, MD, Jennifer Cummings, MD, Mohammed Almualim, MD, and Joseph Atallah, MD

From: University of Toledo Health Science Center, Toledo,

$\mathrm{OH}$

Address Correspondence: Gassan M. Chaiban, MD University of Toledo Medical Center

Department of Anesthesiology University of Toledo, MS 1137 3000 Arlington Avenue Toledo, $\mathrm{OH} 43614$ Email:

gassan.chaiban@utoledo.edu

Conflict of interest: Each author certifies that he or she, or a member of his or her immediate

family, has no commercial association, (i.e., consultancies, stock ownership, equity interest, patent/licensing arrangements, etc.) that might post a conflict of interest in connection with the submitted manuscript. Disclaimer: Spinal Cord Stimulator donated by Boston Scientific.

Manuscript received: 02-15-2013 Revised manuscript received: 04-30-2013

Accepted for publication: 05-20-2013

Free full manuscript: www.painphysicianjournal.com
Background: Spinal cord stimulators (SCS) have been used for many years to treat a myriad of chronic pain conditions using electrical signals to diminish the perception of a painful stimulus. Because of the electrical nature of the devices, there is a concern about the potential for electromagnetic interaction between the device and lifesaving cardiac implantable cardiovertersdefibrillators (ICVD).

Objective: The purpose of this study was to use a swine model to evaluate the potential for interaction between an implanted SCS and ICVD using the closest possible proximity, highest stimulation settings, and most sensitive ICVD settings.

Methods: A pig was anesthetized and subsequently an ICVD and ICVD lead (Cogni 100-D and Endotak Reliance ${ }^{\circledR}$, Boston Scientific, Natick, MA) were placed into the right prepectoral region and the right ventricle, respectively. An SCS (50 cm linear ST Precision Plu octad electrode lead [Boston Scientific, Valencia, CA] with $3 \mathrm{~mm}$ wide contacts spaced one $\mathrm{mm}$ apart) was implanted using fluoroscopic guidance into the posterior epidural space. Remote interrogation and programming of the ICVD were performed while the SCS lead was placed in as close proximity as possible, using fluoroscopy to guide the final position of the SCS electrode. After confirming that both systems were working, appropriately 9 stimulating configurations of varying current, pulse width, and frequency, including maximal settings, were delivered through the SCS. The effects on the ICVD were recorded at 2 sensitivity settings.

Results: None of the tested SCS configurations caused interference with the proper functioning of the ICVD.

Limitations: The anatomical proximity of the posterior epidural space and right ventricle of the swine is different from humans. While the entire pacer, including generator, was imbedded in a subcutaneous pocket, an implantable pulse generator for the SCS was not implanted, which did not allow us to study if any damage or a resetting of settings had occurred to the generator. Only one manufacturer was used in this study. Also, this study was performed in an anesthetized pig and the anatomical positions remained static. Realistically, changes in position of the devices would occur in patients who perform activities of daily living, and this can potentially shorten the distance between the 2 leads causing adverse interaction.

Conclusion: This study clearly demonstrated the feasibility of the 2 devices coexisting and functioning appropriately in an animal model using an ICVD and SCS donated by Boston Scientific. Further studies are needed to elucidate restrictions, optimal settings and parameters in a human setting.

Key words: Spinal cord stimulator, interaction, implantable cardioverter defibrillator, pacemaker, swine model, cross-talk.

Pain Physician 2013; 16:489-496 


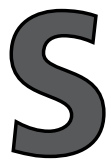
pinal cord stimulators (SCS) were initially introduced for the treatment of chronic pain based on the "gate control" theory with the first unipolar SCS implanted in 1967 (1). They function by transmitting electrical impulses generated from an implanted battery to electrode contacts placed at different locations within the epidural space depending on the painful area to be treated. SCS implantation has been successfully used to treat patients with complex regional pain syndrome, neuropathic pain in the extremities, nonsurgical severe peripheral vascular disease, diabetic neuropathy, failed back surgery syndrome, and chronic angina secondary to ischemic heart disease.

Implanted cardioverter-defibrillators (ICVD) are used to electronically detect and treat potentially lifethreatening cardiac arrhythmias. As the population of patients we treat ages, the incidence of patients with both chronic pain and cardiac disease will increase. Because of the electrical nature of SCS and ICVD, there has been concern that electromagnetic interference or cross-talk between the 2 devices implanted in the same patient may lead to failure of one or both of the systems or inappropriate shocks from the ICVD in response to stimulation from the SCS. The purpose of this study was to demonstrate the possible effects of SCS on ICVD sensing and triggering utilizing a swine animal model.

\section{Methods}

\section{Animal Preparation}

An animal use protocol form was submitted and approved by our institution's Animal Care and Use Committee. All of investigators involved in the study underwent training provided by the Division of Laboratory Animal Resources. The laboratory staff provided an approximately $57 \mathrm{~kg}$ male Yorkshire pig which was acclimated and cared for prior to the procedure in accordance with the standard care and use of laboratory animals protocol at our institution. Prior to the start of the procedure the animal was prepared as follows: ketamine (11-33 $\mathrm{mg} / \mathrm{kg}$ intramuscular) was administered after which vital signs were obtained. The animal was bathed, an IV line established in the ear, and necessary shaving conducted. The animal was intubated with an appropriately sized endotracheal tube and mechanically ventilated with oxygen; isoflurane was administered for anesthetic maintenance. Buprenorphine (0.005-0.01 $\mathrm{mg} / \mathrm{kg}$ intramuscular) was given during this procedure for analgesia. The anesthetic depth was continuously monitored and assessed by laboratory personnel. They determined the depth of the anesthesia by monitoring jaw tone and eye reflexes, and by hoof and skin pinch.

\section{Placement of the ICVD}

A right internal jugular cutdown was performed and a splittable 9 French sheath was advanced into the vein. Through this sheath, an Endotak Reliance ${ }^{\circledR}$ integrated bipolar dual coil defibrillator lead (Boston Scientific, Natick, MA) was advanced. Using fluoroscopic guidance, the lead was advanced into the right ventricle and affixed to an apical position. Impedance, capture, and sensing thresholds were tested and found to be excellent. The sheath was removed and the lead was affixed to the fascial tissue using 2 silk sutures. Then a pre-pectoral pocket was created on the right ventral surface of the swine similar to the location in humans. Once the pocket was created, the lead was then tunneled to the pocket and plugged into a Cognis 100-D internal cardiac defibrillator (Boston Scientific, Natick, MA) and the set screws were tightened (Fig. 1). The device was placed in the pocket and the wound was closed. Baseline measurements through the device demonstrated $\mathrm{R}$ wave sensing of $17.1 \mathrm{mV}$ impedance of 530 ohms, and capture threshold of $0.1 \mathrm{~V}$ at 0.5 milliseconds.

\section{Placement of the SCS}

With the pig placed in the left decubitus position, a 14-gauge modified Tuohy needle was percutaneously advanced from the dorsal surface into the epidural space using fluoroscopic guidance in both anteroposterior and lateral views, and by using the loss of resistance to air technique using a glass syringe. Once the epidural space was reached, a single $50 \mathrm{~cm}$ linear ST Precision Plus octad electrode lead with $3 \mathrm{~mm}$ wide contacts spaced $1 \mathrm{~mm}$ apart (Boston Scientific, Valencia, CA) was advanced under fluoroscopic guidance in the posterior epidural space to the area overlying the heart closest in physical proximity to the intracardiac defibrillator lead (Figs. 2,3). The lead was then connected to an external generator provided by Boston Scientific. The stimulation was programmed using a patient programmer via infrared technology. There were 9 different settings programmed into the stimulator electrode with varying currents, pulse widths, and frequencies up to the maximum allowable settings (Table 1 ).

\section{Stimulation and detection of interference}

For this study, sensing of the ICVD was programmed 


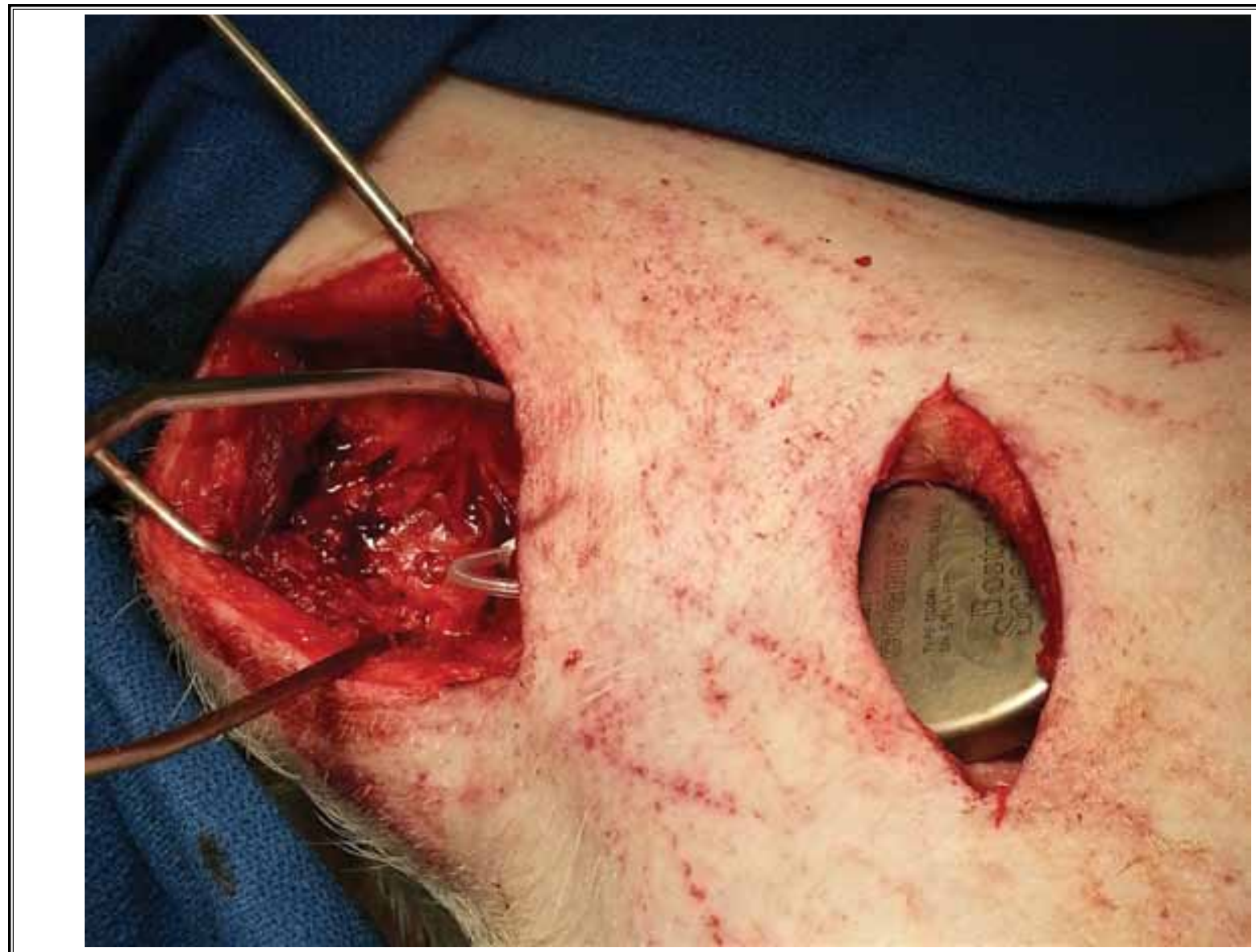

Fig. 1. ICD embedded in pocket prior to closing skin.
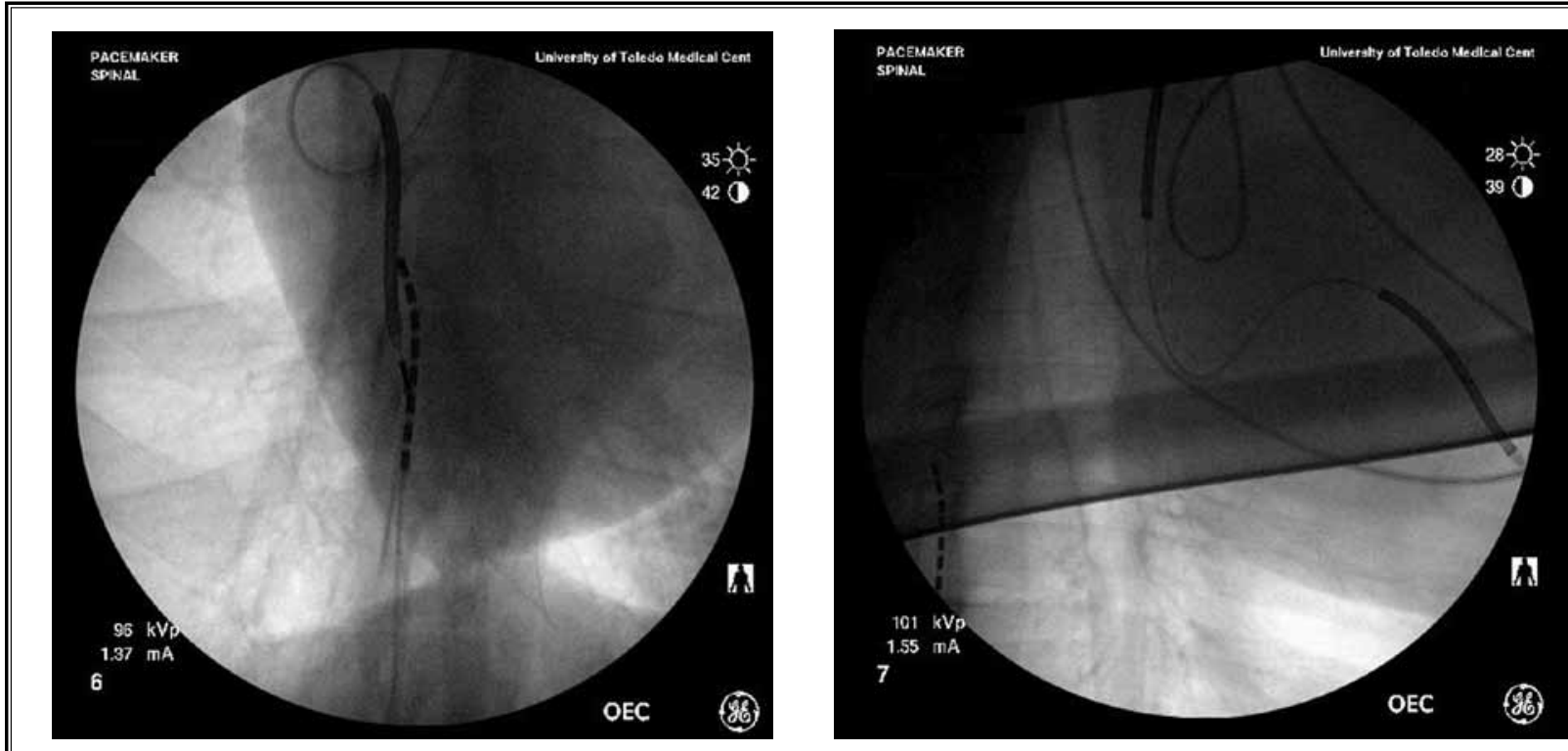

Fig. 2. AP view and Lateral view of final lead position. 


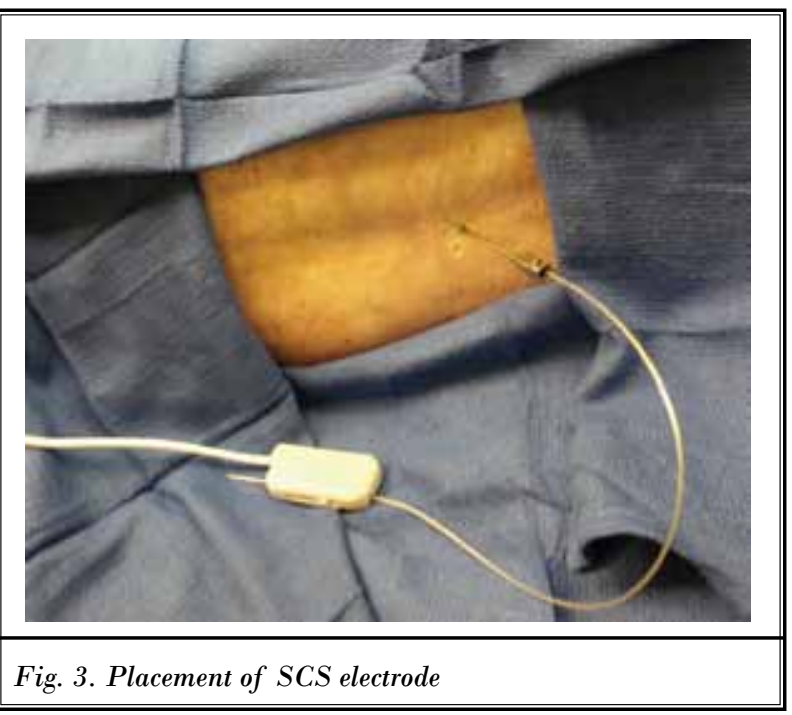

Table 1. Settings, currents, frequencies of SCS.

\begin{tabular}{|c|c|c|c|c|}
\hline Setting & $\begin{array}{c}\text { Current } \\
(\mathbf{m A})\end{array}$ & $\begin{array}{c}\text { Pulse Width } \\
(\mathbf{m s})\end{array}$ & $\begin{array}{c}\text { Frequency } \\
(\mathbf{H z})\end{array}$ & $\begin{array}{c}\text { Interference } \\
(\text { Yes/No) }\end{array}$ \\
\hline 1 & 1 & 210 & 40 & No \\
\hline 2 & 4 & 210 & 40 & No \\
\hline 3 & 4 & 500 & 40 & No \\
\hline 4 & 6.5 & 800 & 40 & No \\
\hline 5 & 10 & 1000 & 40 & No \\
\hline 6 & 12.7 & 1000 & 100 & No \\
\hline 7 & 12.7 & 1000 & 130 & No \\
\hline
\end{tabular}

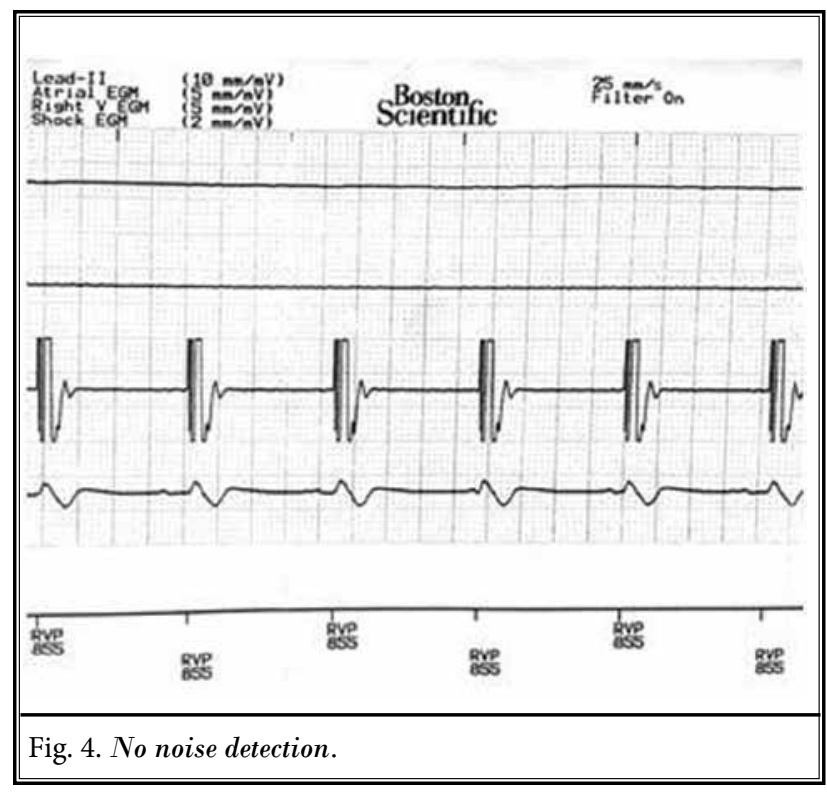

and evaluated at 2 different sensitivity settings during 9 different spinal stimulator settings. The first setting was a sensitivity of $0.6 \mathrm{mV}$ which is what the device would be programmed at in vivo based on implant measurements. The second setting of $0.15 \mathrm{mV}$ was the most sensitive setting available on our model ICVD. The duration of stimulation was approximately one minute for each setting, which allowed time for recording of the electrocardiogram, and printing of a copy for further evaluation.

Remote wireless interrogation of the device was done continuously. During each of the 9 stimulator settings a strip was recorded showing the rate-sensing bipolar sensing channel and the marker channel to show if any cross stimulator "noise" was recorded and/ or inappropriately detected by the ICVD at $0.6 \mathrm{mV}$ and $0.15 \mathrm{mV}$ sensitivity.

\section{RESULTS}

There was no noise visually seen or detected by the device at any of the above settings. Figure 4 demonstrates appropriate pacing with no oversensing, inhibition or noise detection through the ICVD set at its most sensitive level $(0.15 \mathrm{mV})$ during spinal stimulation at a pulse width of 180 microseconds, frequency of $1100 \mathrm{~Hz}$ and current of $2.2 \mathrm{~mA}$. Additionally, while undergoing spinal stimulation the swine spontaneously developed ventricular fibrillation during which the device appropriately detected and shocked into a ventricular paced rhythm. This demonstrated appropriate sensing of ventricular fibrillation by the device at the most sensitive setting while undergoing significantly high spinal stimulation settings. The stimulating electrode was not adversely affected by the ICVD discharge. The impedance readings remained in normal range, and stimulation continued uninterrupted.

\section{Discussion}

Implantable electrical devices are becoming increasingly common treatment modalities for a variety of conditions. These devices include cardiac and gastric pacemakers, ICVDs, insulin pumps, deep brain stimulators (DBS), intrathecal drug delivery pumps and pain neuromodulators. As the utility of these devices continues to expand, it becomes more likely that as our average lifespan lengthens multiple devices will be implanted within the same patient. There has been extensive work reported in the literature regarding external electromagnetic interference (EMI) on these devices, and EMI between multiple devices in the same 
patient (2-7). While there have been many case reports describing the successful use of multiple devices in the same patient, there are some reports of EMI causing disruption of the normal functioning of a device which keeps us questioning not only whether it really is safe to place multiple devices in the same patient, but if you do, what is the safest way to do it?

One of the first publications to report a case series of 10 patients implanted with a permananent Pacemenr (PPM) and SCS was by Romano M et al (4). They discussed one case that revealed intermittent inhibition of a pacemaker with increasing stimulation amplitudes from a spinal cord stimulator, while the other 9 patients had no problems. Based on that study the use of multiple devices in the same patient could not be recommended (8). A review of the simultaneous use of neurostimulators with cardiovascular implanted electronic devices by Ooi et al (1) suggested that PPMs and neurostimulation (NS) can be safely used together. They reviewed 17 studies which revealed 57 uniquely published cases, 41 in which SCS and PPM were used simultaneously. There was only the single case from Romano et al (4) which showed any evidence of interaction (1). In contrast to the use of PPM and SCS simultaneously, there are fewer case reports on the coexistence of SCS and ICVDs.

Our MEDLINE search revealed 13 studies which included 22 unique cases of patients who have an ICVD and either SCS or DBS (1,5,7,9-17) (Table 2). Two of the cases had adverse outcomes. There is a case report by Tavernier et al (5) of a total reset of the electrode polarities and resetting of the output state to off in 2 pectorally implanted pulse generators of a DBS. This occurred after an appropriately sensed arrhythmia and discharge from an abdominally placed ICVD. In this case the functioning of the ICVD was not affected by the presence of the stimulators, but the electrical discharge from the internal defibrillation had a significant effect on the programming of the DBS pulse generators (5). In the case series presented by Molon et al (13) one patient experienced SCS power reset due to multiple ICVD shocks caused by T-wave oversensing. None of the other cases reported ICVD discharge during testing. The functioning of both systems was evaluated and no cross-talk between the devices was described despite having used maximum stimulation settings and maximum sensitivities on the ICVDs (13).

Our study used both an ICVD and SCS provided by Boston Scientific. In our literature review none of the cases that we found included both an ICVD and SCS manufactured by Boston Scientific. The cases that we found were predominantly devices manufactured by Medtronic.

The Web sites of the 3 major neurostimulator manufacturers (Boston Scientific, Medtronic, and St. Jude) have warnings about using ICVDs and SCS simultaneously. Medtronic's Web site warns, "Sources of strong electromagnetic interference (e.g., defibrillation, diathermy, electrocautery, [magnetic resonance imaging] MRI, [radiofrequency] RF ablation, and therapeutic ultrasound) can interact with the neurostimulation system, resulting in serious patient injury or death. These and other sources of EMI can also result in system damage, operational changes to the neurostimulator or unexpected changes in stimulation" (7). It also mentions the presence of ICVDs stating, "An implanted cardiac device (e.g., pacemaker, defibrillator) may damage a neurostimulator, and the electrical pulses from the neurostimulator may result in an inappropriate response of the cardiac device" (7). The Boston Scientific Web site states, "Spinal cord stimulators may interfere with the operation of implanted sensing stimulators such as pacemakers or cardioverter defibrillators. The effects of implanted stimulation devices on neurostimulators are unknown" (18). For their neurostimulators, the St. Jude Web site states, "The system is contraindicated for patients with demand-type cardiac pacemakers." It also provides the warning that, "neurostimulation systems may adversely affect the programming of implanted cardioverter defibrillators" (19).

Based on our literature search, there have not been any published prospective trials that studied the interaction between an ICVD and SCS implanted in the same patient. An animal model can closely approximate the anatomical locations in a state that is physiologically equivalent to a human model. In this study we actively attempted to cause interference between the 2 devices by placing the leads of both devices in as close an anatomical relationship as possible. We increased the settings of the stimulating electrode to generate as much current as possible, and set the ICVD to be as sensitive as possible to maximize vulnerability for interference between the 2 devices. We observed for signs of over- or under-sensing and the possibility of leading to inappropriate shocks. None of the settings that were programmed into the SCS caused interference or inappropriate shocks from the ICVD, indicating that both may be safely used in the same body. Additionally, the ICVD appropriately detected ventricular fibrillation which it successfully defibrillated with 36 joules when the anesthetized swine spontaneously developed ven- 
Table 2. Cases and studies relevant to SCS research.

\begin{tabular}{|c|c|c|c|c|c|c|}
\hline $\begin{array}{l}\text { Author, year } \\
\text { published }\end{array}$ & $\begin{array}{l}\text { \# of cases } \\
\text { reported }\end{array}$ & $\begin{array}{c}\text { neuro } \\
\text { stimulation type }\end{array}$ & $\begin{array}{l}\text { Level of } \\
\text { SCS leads }\end{array}$ & $\begin{array}{c}\text { Stimulator make and } \\
\text { model }\end{array}$ & $\begin{array}{c}\text { AICD make and } \\
\text { model }\end{array}$ & Interference \\
\hline A.Katwal 2009 & 1 & SCS & $\mathrm{C} 7$ & ITREL 3, 7425 Medtronic & $\begin{array}{c}\text { Medtronic, Vertuoso } \\
\text { D154AWG }\end{array}$ & No \\
\hline R.Schimpf 2003 & 1 & SCS & T11 & ITREL 3, 7425 Medtronic & $\begin{array}{l}\text { GEM 3, AT } 7276 \\
\text { Medtronic }\end{array}$ & No \\
\hline $\begin{array}{l}\text { K.Monahan } \\
1998\end{array}$ & 1 & SCS & T10 & ITREL 3, 7425 Medtronic & $\begin{array}{l}\text { Medtronic } 7221 \mathrm{gx} \\
\text { active can }\end{array}$ & No \\
\hline J.Rosenow 2003 & 1 & DPS & BSTN & Medtronic, Model 6947 & Isoletra ,Medtronic & No \\
\hline $\begin{array}{l}\text { A.Obwegeser } \\
2001\end{array}$ & 1 & DPS & UVIN & Medtronic, model 3387 & GEM DR 7271 model & No \\
\hline $\begin{array}{l}\text { R Tavernier } \\
2000\end{array}$ & 1 & DPS & BSTN & $\begin{array}{c}\text { ITREL } 37425,7221 \mathrm{D} \\
\text { Medtronic }\end{array}$ & Medtronic 6945 & $\begin{array}{l}\text { Reset of the DBS } \\
\text { after ICD shock }\end{array}$ \\
\hline \multirow{2}{*}{ A.Sharan 2010} & 2 & SCS & T10-11 & ITREL 2, Medtronic & Medtronic, Virtuoso & No \\
\hline & & DBS & BSTN & Kinetra, Medtronic & Medtronic, Concerto & No \\
\hline H.Dorman 2011 & 1 & SCS & & $\begin{array}{c}\text { Synergy, versitrel } \\
\text { Medtronic }\end{array}$ & $\begin{array}{c}\text { Consulta D234TRK } \\
\text { medtronic } \\
\end{array}$ & No \\
\hline \multirow{5}{*}{$\begin{array}{l}\text { T.Enggaard } \\
2009\end{array}$} & \multirow{5}{*}{5} & SCS & $\mathrm{C} 7-\mathrm{T} 1$ & ITREL 3, Medtronic & Guidant/ Medtronic & No \\
\hline & & SCS & C7-T1 & ITREL 3, Medtronic & Guidant/ Medtronic & No \\
\hline & & SCS & C7-T1 & ITREL 3, Medtronic & Guidant & No \\
\hline & & SCS & C7-T1 & ITREL 3, Medtronic & Medtronic & No \\
\hline & & SCS & $\mathrm{C} 7-\mathrm{T} 1$ & Synergy, Medtronic & Medtronic & No \\
\hline \multirow{3}{*}{ G. Molon 2011} & \multirow[t]{3}{*}{3} & SCS & & ITREL 3, 7425 Medtronic & Intrinsic ,Model 7288 & $\begin{array}{c}\text { Temporary } \\
\text { damage to SCS } \\
\text { after multiple ICD } \\
\text { discharges at } 35 \mathrm{~J} \\
\end{array}$ \\
\hline & & $\begin{array}{c}\text { Sacral } \\
\text { neurostimulator }\end{array}$ & $\begin{array}{l}\text { 3rd sacral } \\
\text { foramen }\end{array}$ & $\begin{array}{c}\text { InterStim, Model } 3023 \\
\text { Medtronic }\end{array}$ & Consulta, Medtronic & No \\
\hline & & SCS & & ITREL 3, 7425 Medtronic & Consulta, Medtronic & No \\
\hline $\begin{array}{l}\text { S.Eckert } 2009 \\
\text { (abstract) }\end{array}$ & 5 & & & & & No \\
\hline
\end{tabular}

AICD:Automated implantable cardioverters-defibrillators. SCS: Spinal cord stimulator. C: Cervical. T: Thoracic. BSTN: Bilateral subthalamic nucleus. UVIN: Unilateral ventral intermediate nucleus. DBS: Deep brain stimulators.

tricular arrhythmias while the stimulator was actively being used.

The limitations of this study include that the anatomical proximity of the posterior epidural space and right ventricle of the swine are different from humans. While the entire pacer, including generator, was imbedded in a subcutaneous pocket, an implantable pulse generator for the SCS was not implanted, but remained outside the body. This did not allow us to study if any damage or a resetting of settings would occur to the generator as had happened in the 2 cases mentioned previously. We only used one manufacturer in this study to ensure that the ICVD and stimulator were from the same company. In practice, this is not always the case. There are multiple manufacturers of ICVDs, pacemakers, and stimulators, and the possibility of interactions between different device companies or different models of devices was not addressed in this study. This may be an area to study in the future. The duration of time that we performed stimulation at each of the settings was brief (approximately one minute); this limitation does not help address the question of 
duration of stimulation. In the future the possibility of a longitudinal study where the effects of placement of multiple devices is evaluated after several months or a year after implantation should be considered. Finally, this study was performed in an anesthetized pig and the anatomical positions remained static. Realistically, changes in position of the devices will occur in patients who perform activities of daily living, and this can potentially shorten the distance between the 2 leads, causing adverse interaction. Case reports have tested the compatibility between an ICVD and SCS during position changes by the patient as part of their algorithm for determining compatibility (9). In our study we positioned the electrodes of the SCS as close as anatomically possible to the sensing portion of the defibrillator lead. We do not know if placing the leads in closer proximity to the ICVD generator would have caused interference. This may become a concern in cases where peripheral nerve field stimulation occurs as in the treatment for occipital neuralgia, when the generator for the ICVD and the stimulating electrodes may both be embedded in the subcutaneous tissue in close proximity to each other (20). The stimulation settings were also incrementally increased in our study, whereas in use, patients randomly switch between settings that can be drastically different. They can also turn their stimulator on and off at will.

There have been many case reports which describe the safe implementation of both PPM and SCS in patients. Only the St. Jude Web site had a specific contraindication to the placement of a demand pacemaker and neurostimulator (19). The other 2 companies did not list specific contraindications on the use of both simultaneously, but general caution and frequent assessment has been advocated in the literature to ensure proper functioning of both systems $(1,21)$. The same appears to hold true for the placement of an ICVD and SCS, where none of the case reports indicated any interference with the functioning of the lifesaving ICVD, even though there are 2 reports of ICVD discharge resetting the stimulators. In our opinion, previously proposed recommendations to avoid potential interaction should continue to be followed. These include putting the 2 devices in op- posite sides of the body, programming both systems to be in bipolar configurations, and ventricular fibrillation testing of the ICVD should be immediately performed (1). Studies have provided recommendations for programming the SCS to bipolar configuration with a pulse width as low as possible and a frequency higher than 60 $\mathrm{Hz}$, to reduce the risk of interaction (13). Intraoperative and immediate postoperative tests combining different configurations, even if it raises the parameters of the stimulator to an uncomfortable level for the patient, can be done just to confirm whether some values might give rise to interference (13).

Close followup and frequent testing of both systems should continue to occur after placement. Schimpf et al (9) postulated that during the life of an electrode, possible complications might occur such as fracture, migration, or insulation failure that could generate leakage of current which could be detected and inappropriately treated by the ICVD. This would continue to necessitate that patients with 2 or more implanted electrical devices be carefully assessed to detect any potential interactions early on during implantation. Also, after the implantation of SCS, fibrosis around the electrode can cause the impedance to change by up to $26 \%$ by 3 months after implantation (22). This can require changing the settings and direction of current in order to successfully continue capture of all painful areas. This mandates short follow-up time intervals after implantation with the suggested evaluation of all implanted systems once per month for the first 3 months, followed by every 3 months for the first year, and then every 6 months thereafter. Additionally, if any changes are required to the devices, they should be rechecked for the possibility of interaction.

\section{Conclusion}

In conclusion, this study clearly demonstrated the feasibility of the 2 devices coexisting and functioning appropriately in an animal model using an ICVD and SCS provided by Boston Scientific. Further studies are needed to elucidate restrictions, optimal settings, and parameters in a human setting. 


\section{References}

1. Ooi YC, Falowski S, Wang D, Jallo J, Ho RT, Sharan A. Simultaneous use of neurostimulators in patients with a preexisting cardiovascular implantable electronic device. Neuromodulation 2011; 14:20-26.

2. Hayes DL, Wang PJ, Reynolds DW, Estes M 3rd, Griffith JL, Steffens RA, Carlo GL, Findlay GK, Johnson CM. Interference with cardiac pacemakers by cellular telephones. N Engl J Med 1997; 336:1473-1479.

3. Pinski SL, Trohman RG. Interference in implanted cardiac devices, Part I. Pacing Clin Electrophysiol 2002; 25:1367-1381.

4. Romanó M, Zucco F, Baldini MR, Allaria B. Technical and clinical problems in patients with simultaneous implantation of a cardiac pacemaker and spinal cord stimulator. Pacing Clin Electrophysiol 1993; 16:1639-1644.

5. Tavernier R, Fonteyne W, Vandewalle $V$, de Sutter J, Gevaert S. Use of an implantable cardioverter defibrillator in a patient with two implanted neurostimulators for severe Parkinson's disease. Pacing Clin Electrophysiol 2000; 23:1057-1059.

6. Monahan K, Casavant D, Rasmussen C, Hallet N. Combined use of a true-bipolar sensing implantable cardioverter defibrillator in a patient having a prior implantable spinal cord stimulator for intractable pain. Pacing Clin Electrophysiol 1998; 21:2669-2672.

7. Medtronic Spinal Cord Stimulation System http://professional.medtronic.com/ pt/neuro/scs/ind/index.htm

8. Kosharskyy B, Rozen D. Feasibility of spinal cord stimulation in a patient with a cardiac pacemaker. Pain Physician
2006; 9:249-251.

9. Schimpf R, Wolpert C, Herwig $S$, Schneider C, Esmailzadeh B, Lüderitz B. Potential device interaction of a dual chamber implantable cardioverter defibrillator in a patient with continuous spinal cord stimulation. Europace 2003; 5:397-402.

10. Thomas P. Enggaard, Claus Andersen, and Christian Scherer. Spinal cord stimulation for refractory angina in patients implanted with cardioverter defibrillators: Five case reports. Europace 2010; 12:1336-1337.

11. Dorman H.G, Onrust A, Brants R, Stevenhagen YJ . Scholten M.F. Use of a biventricular implantable cardioverter defibrillator in a patient with neurostimulator: Is it a safe combination? Neth Heart] 2011; April 13: Epub ahead of print.

12. Eckert S, Horstkotte D. Management of angina pectoris: The role of spinal cord stimulation. Am J Cardiovasc Drugs 2009; 9:17-28.

13. Molon G, Perrone C, Maines M, Costa A, Comisso J, Boi A, Moro E, Vergara G, Barbieri E. ICD and neuromodulation devices: Is peaceful coexistence possible? Pacing Clin Electrophysiol 2011; 34:6:690-693.

14. Rosenow JM, Tarkin H, Zias E, Sorbera C Mogilner A. Simultaneous use of bilateral subthalamic nucleus stimulators and an implantable cardiac defibrillator. Case report. J Neurosurg 2003; 99:167-169.

15. Obwegeser AA, Uitti RJ, Turk MF, Wszolek UM, Flipse TR, Smallridge RC, Witte RJ, Wharen RE Jr. Simultaneous thalamic deep brain stimulation and implantable cardioverter-defibrillator. Mayo Clin Proc 2001; 76:87-89.
16. Katwal $A B$, McCotter C). Use of a wireless implantable cardioverter defibrillator in a patient with a preexisting neurostimulator. Pacing Clin Electrophysiol 2009; 32:822-824.

17. Ferrero $P$, Grimaldi R, Massa R, Chiribiri A, De Luca A, Castellano M, Cardano P, Trevi GP. Spinal cord stimulation for refractory angina in a patient implanted with a cardioverter defibrillator. Pacing Clin Electrophysiol. 2007; 30:143-146.

18. Boston Scientific: Precision Plus TM Spinal Cord Stimulator System. http://www.bostonscientific.com/ Device.bsci ?page $=$ ResourceDetail \& navRelld $=1000.1003 \&$ method $=$ DeD etailHCP\&id=10068931\&resource type_category_id=1\&resource_type_ $i d=91$ \&pageDisclaimer=Disclaimer. ProductPage

19. The St. Jude EonC TM Primary Cell IPG System http://www.sjmneuropro.com/ Products/US/EonC-Primary-Cell-IPGSystem.aspx

20. Kapural L, Sable J. Peripheral nerve stimulation for occipital neuralgia: Surgical leads. Prog Neurol Surg 2011; 24:86-95.

21. Ekre O, Börjesson M, Edvardsson N, Eliasson T, Mannheimer C. Feasibility of spinal cord stimulation in angina pectoris in patients with chronic pacemaker treatment for cardiac arrhythmias. Pacing Clin Electrophysiol 2003; 26:2134-2141.

22. Kreis PG, Fishman SM. Spinal cord stimulation, percutaneous implantation techniques. Oxford University Press, New York, 2009. 